\title{
Moment formulation for random eigenvalue problems in beams
}

\author{
B. W. Yeigh ${ }^{1} \&$ J. A. Hoffman ${ }^{2}$

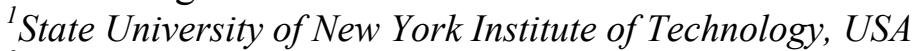 \\ ${ }^{2}$ Engineering Solutions, LLC, USA
}

\begin{abstract}
This paper proposes a moment formulation model to handle eccentric load imperfections in beams on elastic foundation taking into consideration the randomness of imperfections. An alternative approach to the secant formula to capture the effects of load imperfections is described. The study demonstrates eccentricity is the most detrimental form of structural imperfections and that it aggressively and adversely interacts with other imperfections.

Keywords: stability, load imperfections, beam on elastic foundation, regular perturbation, eigenvalue, energy method, stochastic, spectral representation.
\end{abstract}

\section{Introduction}

One fundamental problem still remains to be explored in structural engineering are the stability of imperfection sensitive structures. Unlike tension and flexural members that fail when the applied loads cause stresses that exceed material limitations, slender columns most often fail by buckling. Furthermore, column buckling does not depend on the proportional limit of the member. Buckling is a complex failure mechanism that is often catastrophic with little or no warning. It depends not only on the material and section properties of the column, but also on the contributions and interactions of its length, end support conditions, lateral supports, and location of the applied load.

The classical stability analysis $[5,7]$, is actually developed largely from the work of Leonhard Euler who first analytically investigated the column buckling phenomenon in 1744. Over the years, much has been done to extend and refine Euler's work. Buckling formulations based on the Euler equation had some rational consideration for the behavior of the material in question. They all 
perform empirically well for ideal columns with concentric loads, and models that are not unusually sensitive to imperfections. However, imperfections exist and are common in real structures. In this study, the authors define structural imperfections as any small, unavoidable deviations from the perfect structure. These deviations include those of shape (i.e., initial curvature), material properties, section properties, support mechanisms, and the geometric configuration of the applied load such as accidental eccentricity.

While not all structures are sensitive to imperfections, experience and experimentation have shown that some structures including the beam on elastic foundation and cylindrical shells are quite sensitive to structural imperfections [8]. One way to address imperfections is to design structures by increasing factors of safety. For the most part, engineers approach imperfections by using some rational means to determine the stresses in a column that are caused by the imperfection, and then checking this against the Euler load, possibly with an added safety factor. In an attempt to more accurately analyze the effect of shape imperfections, the secant formula is used to capture the effects of the imperfection [6]. While the secant formula will give good results for the stresses in a column with imperfections, it is not a buckling formula. It is a strength formula. The formula gives an excellent estimate of the stress in the column, but it does not and can not predict whether or not the column has survived up to the classical buckling load, nor does it say anything about imperfection sensitivity.

However, if a structure is sensitive to imperfections, an unstable equilibrium exists at the critical load, and it is possible for neighboring equilibria to exist at loads less than the critical load. The structure may experience structural "softening" as it nears the critical load and consequently less load is required to produce more deflection [1]. Examples of structures that are sensitive to structural imperfections are thin shells, space frames, thin-walled beams, arches, and laterally supported columns such as the beam on elastic foundation (BEF). This paper considers eccentricities in the applied loads in BEF.

\section{Numerical method}

Introduced first by Palassopoulos [3], the Critical Imperfection Magnitude Method (CIM) is a robust regular perturbation method that addresses limitations of Koiter's method [2]. CIM considers all types of imperfections and not just shape imperfections. Yeigh [9] provided a stochastic interpretation of the method. CIM takes the potential energy to the second-order expansion and kinematically admissible set of generalized coordinates to the fourth-order expansion. The general method is outlined in Palassoupoulos [3], and stochastic formulation is presented in Yeigh [9]. The potential energy of the "perfect" structure (i.e., no imperfections), $\mathrm{V}_{0}$, is expanded in terms of the generalized coordinates $\mathrm{q}_{\mathrm{j}}$, where $\mathrm{j}=1,2, \ldots, \mathrm{M}$.

$$
\mathrm{V}_{0}=\mathrm{v}_{0}+\mathrm{a}_{0 \mathrm{j}} \mathrm{q}_{\mathrm{j}}+\mathrm{b}_{0 \mathrm{jk}} \mathrm{q}_{\mathrm{j}} \mathrm{q}_{\mathrm{k}}+\mathrm{c}_{0 \mathrm{jkl}} \mathrm{q}_{\mathrm{j}} \mathrm{q}_{\mathrm{k}} \mathrm{q}_{1}+\mathrm{d}_{0 \mathrm{jklm}} \mathrm{q}_{\mathrm{j}} \mathrm{q}_{\mathrm{k}} \mathrm{q}_{1} \mathrm{q}_{\mathrm{m}}+\ldots
$$

Then the potential energy of the "actual" structure (i.e., with imperfections), $\mathrm{V}$ is discretized and expanded: 


$$
\begin{gathered}
\mathrm{V}=\mathrm{V}_{0}+\varepsilon \mathrm{V}_{1}+\varepsilon^{2} \mathrm{~V}_{2}+\ldots \\
\mathrm{V}_{1}=\mathrm{v}_{1}+\mathrm{a}_{1 \mathrm{j}} \mathrm{q}_{\mathrm{j}}+\mathrm{b}_{1 \mathrm{jk}} \mathrm{q}_{\mathrm{j}} \mathrm{q}_{\mathrm{k}}+\mathrm{c}_{1 \mathrm{jk} \mathrm{l}} \mathrm{q}_{\mathrm{j}} \mathrm{q}_{\mathrm{k}} \mathrm{q}_{1}+\ldots \\
\mathrm{V}_{2}=\mathrm{v}_{2}+\mathrm{a}_{2 \mathrm{j}} \mathrm{q}_{\mathrm{j}}+\mathrm{b}_{2 \mathrm{jk}} \mathrm{q}_{\mathrm{j}} \mathrm{q}_{\mathrm{k}}+\mathrm{c}_{2 \mathrm{jk} \mathrm{k}} \mathrm{q}_{\mathrm{j}} \mathrm{q}_{\mathrm{k}} \mathrm{q}_{1}+\ldots
\end{gathered}
$$

where the coefficients $\mathrm{a}_{(.)}, \mathrm{b}_{(.)}, \mathrm{c}_{(.)}$and $\mathrm{d}_{(.)}$are chosen to be symmetric with respect to permutation of their indices. The universal imperfection magnitude parameter $\varepsilon$ is a measure of the magnitude of deviation in material and structural properties from the perfect structure. In general, any property $\mathrm{S}$ can be modeled as $\mathrm{S}(\mathrm{x})=$ $\mathrm{S}_{0}[1+\varepsilon \mathrm{s}(\mathrm{x})]$ with a mean value of $\mathrm{S}_{0}$ and an imperfection pattern $\mathrm{s}(\mathrm{x})$. When $\varepsilon=$ 0 , the structure is reduced to the perfect structure. However, the product of the imperfection being considered and the critical imperfection magnitude $\varepsilon_{\text {cr }}$ must be sufficiently small $(<0.35)$ in order for the power series expansion to converge. The potential energy can be rewritten in more general form:

$$
\begin{gathered}
\mathrm{V}=\left(\mathrm{v}_{0}+\varepsilon \mathrm{v}_{1}+\varepsilon^{2} \mathrm{v}_{2}+\ldots\right)+\left(\mathrm{a}_{0 \mathrm{j}}+\varepsilon \mathrm{a}_{1 \mathrm{j}}+\varepsilon^{2} \mathrm{a}_{2 \mathrm{j}}+\ldots\right) \mathrm{q}_{\mathrm{j}} \\
+\left(\mathrm{b}_{\mathrm{ojk}}+\varepsilon \mathrm{b}_{1 \mathrm{jk}}+\varepsilon^{2} \mathrm{~b}_{2 \mathrm{jk}}+\ldots\right) \mathrm{q}_{\mathrm{j}} \mathrm{q}_{\mathrm{k}} \\
+\left(\mathrm{c}_{0 \mathrm{jkl}}+\varepsilon \mathrm{c}_{1 \mathrm{jkl}}+\varepsilon^{2} \mathrm{c}_{2 \mathrm{jkl}}+\ldots\right) \mathrm{q}_{\mathrm{j}} \mathrm{q}_{\mathrm{k}} \mathrm{q}_{1}+\ldots
\end{gathered}
$$

The first and second variations offer equilibrium and stability conditions:

$$
\begin{gathered}
\delta \mathrm{V}=\left\{\left(\mathrm{a}_{0 \mathrm{j}}+\varepsilon \mathrm{a}_{1 \mathrm{j}}+\varepsilon^{2} \mathrm{a}_{2 \mathrm{j}}+\ldots\right)\right. \\
+2\left(\mathrm{~b}_{0 \mathrm{jk}}+\varepsilon \mathrm{b}_{1 \mathrm{jk}}+\varepsilon^{2} \mathrm{~b}_{2 \mathrm{jk}}+\ldots\right) \mathrm{q}_{\mathrm{k}} \\
+3\left(\mathrm{c}_{\mathrm{ojkl}}+\varepsilon \mathrm{c}_{1 \mathrm{jkl}}+\varepsilon^{2} \mathrm{c}_{2 \mathrm{jkl}}+\ldots\right) \mathrm{q}_{\mathrm{k}} \mathrm{q}_{1} \\
\delta^{2} \mathrm{~V}=\left\{2\left(\mathrm{~b}_{\mathrm{ojk}}+\varepsilon \mathrm{b}_{1 \mathrm{jk}}+\varepsilon^{2} \mathrm{~b}_{2 \mathrm{jk}}+\ldots\right)\right. \\
+6\left(\mathrm{c}_{\mathrm{ojkl}}+\varepsilon \mathrm{c}_{1 \mathrm{jkl}}+\varepsilon^{2} \mathrm{c}_{2 \mathrm{jkl}}+\ldots\right) \mathrm{q}_{1}
\end{gathered}
$$

Then the eigenvalue problem can be formulated:

$$
\left[\begin{array}{cc}
\gamma_{1} & \gamma_{2} \\
I & 0
\end{array}\right]\left\{\begin{array}{c}
\delta q \\
\varepsilon \delta q
\end{array}\right\}=\frac{1}{\varepsilon}\left\{\begin{array}{c}
\delta q \\
\varepsilon \delta q
\end{array}\right\}
$$

where I and 0 represent identity and zero submatrices.

$$
\begin{gathered}
\gamma_{1 j k}=\frac{1}{\sqrt{b_{0 j j} b_{0 k k}}}\left[-b_{1 j k}+\frac{3 c_{0 j k l} a_{1 l}}{2 b_{0 l l}}\right] \\
\gamma_{2 j k}=\frac{1}{\sqrt{b_{0 j j} b_{0 k k}}}\left[-b_{2 j k}+\frac{3 c_{1 j k l} a_{1 l}}{2 b_{0 l l}}+\frac{3 c_{0 j k l} a_{2 l}}{2 b_{0 l l}}-\frac{3 c_{0 j k l} b_{1 l m} a_{1 m}}{2 b_{0 l l} b_{0 m m}}\right. \\
\left.-\frac{3 c_{0 j k l} b_{1 l m} a_{1 m}}{2 b_{0 l l} b_{0 m m}}+\frac{9 c_{0 j k l} c_{0 l m m} a_{1 m} a_{1 n}}{8 b_{0 l l} b_{0 m m} b_{0 n n}}-\frac{3 d_{0 j k l m} a_{1 l} a_{1 m}}{2 b_{0 l l} b_{0 m m}}\right]
\end{gathered}
$$




\section{Beam on elastic foundation (BEF) formulation with random load imperfections (eccentricity)}

Eccentricity in the applied load can be modeled as a shape imperfection because the resulting end moments induce curvature into the beam. The eccentricity imperfections $\eta_{1}$ and $\eta_{2}$ will be modeled as single-value random variables. The imperfection patterns are assumed to be one-dimensional, homogenous, Gaussian random fields. The cosine series formula [4] will be used to simulate random imperfection patterns. The function $\mathrm{s}(\mathrm{x})$ represents the imperfection pattern which is a $1 \mathrm{D}-1 \mathrm{~V}$, homogeneous, Gaussian stochastic field with a zero mean. In terms of the cosine series with deterministic amplitude $A_{n}$, and a random phase angle $\varphi_{\mathrm{n}}$, the pattern is written as:

$$
\begin{gathered}
s(x)=\sqrt{2} \sum_{n=1}^{N} A_{n} \cos \left(\kappa_{n} x+\varphi_{n}\right) \\
A_{n}=\left(G_{f f}\left(\kappa_{n}\right) \Delta \kappa\right)^{\frac{1}{2}} \\
\kappa_{n}=n \Delta \kappa=n \frac{\kappa_{u}}{N}, \mathrm{n}=1,2, \ldots, \mathrm{N}
\end{gathered}
$$

where $\kappa$ is the wave number and $\kappa_{\mathrm{u}}$ is the fixed upper cut-off wave number. The value of $\kappa_{\mathrm{u}}$ is chosen such that above it, the corresponding one-sided power spectral density $G_{f f}(\kappa)$ is zero or negligibly small. The following power spectral density (PSD) function and corresponding autocorrelation function $\mathrm{R}_{\mathrm{ff}}(\xi)$ are used:

$$
\begin{gathered}
G_{f o f o}\left(\kappa_{0}\right)=\frac{1}{2} \sigma_{f o}^{2} b_{f o}^{3} \kappa_{o}^{2} \exp \left[-b_{f o}\left|\kappa_{o}\right|\right] \\
R_{f o f o}\left(\xi_{0}\right)=\sigma_{f o}^{2} \frac{1-3\left(\frac{\xi_{o}}{b_{f o}}\right)^{2}}{\left[1+\left(\frac{\xi_{o}}{b_{f o}}\right)^{2}\right]^{3}}
\end{gathered}
$$

The correlation distance, $b_{\mathrm{fo}}$, is chosen to best match the PSD to the expected degree of fluctuation in the imperfections. Long values of $b_{f o}$, the PSD undulates slowly. Short values of $b_{f o}$, the PSD varies sharply. The correlation distance used in this study is 1.50 , a reasonable and realistic choice that reflects an actual beam with imperfections.

BEF with imperfections in initial shape, bending rigidity, foundation stiffness, load and eccentric end loads were considered in this study. The beam also has a depth, $d_{e}$. The variable $d_{e}$ does not explicitly enter the potential energy formulation. However, in order to model the distribution of the end 
eccentricities, the depth must be physically defined. This can be achieved by relating $d_{e}$ to length, $L$ by means of a span-to-depth ratio. For this study, a commonly used engineering span-to-depth ratio of 20 is used.

For generality, non-dimensional variables were used throughout. For this problem, the most convenient approach to a non-dimensional form is to divide the length variables by the modified span length, $\mathrm{L}_{\mathrm{P}}=\mathrm{L} / \pi$.

$$
\begin{gathered}
x=\frac{X}{L_{P}}=\frac{\pi X}{L}, w=\frac{W}{L_{P}}=\frac{\pi W}{L}, \varphi=\frac{L_{P}^{4}}{(E I)_{0}} K=\frac{L^{4}}{\pi^{4}(E I)_{0}} K \\
z_{1}=\frac{Z_{1}}{L_{P}}=\frac{\pi Z_{1}}{L}, z_{2}=\frac{Z_{2}}{L_{P}}=\frac{\pi Z_{2}}{L}, \rho=\frac{L^{2}}{\pi^{2}(E I)_{0}} P, v=\frac{L}{\pi(E I)_{0}} V
\end{gathered}
$$

Imperfections in bending rigidity (EI), foundation stiffness $(\mathrm{K})$, load $(\mathrm{P})$, and eccentricity $(Z)$ are described as zero mean stochastic fields:

$$
\begin{gathered}
\mathrm{EI}(\mathrm{x})=(\mathrm{EI})_{0}[1+\varepsilon \mathrm{e}(\mathrm{x})], \varphi(\mathrm{x})=\varphi_{0}[1+\varepsilon \mathrm{k}(\mathrm{x})], \mathrm{w}_{0}=\varepsilon \mathrm{h}(\mathrm{x}) \\
\mathrm{z}_{1}=\varepsilon \eta_{1}, \mathrm{z}_{2}=\varepsilon \eta_{2}, \mathrm{~K}(\mathrm{x})=\mathrm{K}_{0}[1+\varepsilon \mathrm{k}(\mathrm{x})] \\
\varphi_{0}=\frac{L_{P}^{4}}{(E I)_{0}} K_{0}=\frac{L^{4}}{\pi^{4}(E I)_{0}} K_{0}
\end{gathered}
$$

The functions $\mathrm{e}(\mathrm{x}), \mathrm{k}(\mathrm{x})$, and $\mathrm{h}(\mathrm{x})$ are the imperfection patterns (stochastic fields) for the beam to be used on the bending rigidity, foundation stiffness, and initial shape respectively. The terms $\eta_{1}$ and $\eta_{2}$ are independent random variables. For the case of a Gaussian distribution, $\eta$ is distributed normally with zero mean and standard deviation $\sigma_{\eta}$. For physical reasons, the distributions for $\eta$ are truncated at the top and bottom edges of the beam. It is desired to have positively defined end moments $M_{1}$ and $M_{2}$ in the formulation of $V_{M}$. In order for $M_{1}$ to be positive, $Z_{1}$ must be negative. $\left(M_{1}=-P Z 1\right)$ The reverse is true for $M_{2}$. Here, a positive $Z_{2}$ gives a positive $M_{2} .\left(M_{2}=P Z_{2}\right)$

There are four contributing components to the potential energy of the BEF: the strain energy of bending due to change in curvature $\left(V_{B}\right)$, the strain energy of the foundation $\left(\mathrm{V}_{\mathrm{K}}\right)$, the potential energy of the applied load $\left(\mathrm{V}_{\mathrm{P}}\right)$, and the potential energy of the moments resulting from the accidental eccentricities $\left(V_{M}\right): V=V_{B}+V_{K}+V_{P}+V_{M}$. Before proceeding with the development of the coefficients in the characteristic equation, the spectral representations for $\mathrm{e}(\mathrm{x})$, $\mathrm{k}(\mathrm{x})$, and $\mathrm{h}(\mathrm{x})$ must be developed.

$$
e(x)=\sum_{j=1}^{N} e_{j} \cos \left(\kappa_{j}^{e} x+\Phi_{j}^{e}\right) \quad k(x)=\sum_{j=1}^{N} k_{j} \cos \left(\kappa_{j}^{k} x+\Phi_{j}^{k}\right)
$$

A direct representation for $\mathrm{h}(\mathrm{x})$ similar to eqn. (21) is not possible. The cosine series does not work because the forced end conditions require zero end displacements. Addressing the incompatibility question requires first generating the shape imperfection field using the spectral representation method, and then 
rotating and translating the field to meet the required boundary conditions. This fitting procedure is described as follows:

$$
h^{*}(x)=\left[\sqrt{2} \sum_{i=1}^{N} h_{i} \cos \left(\kappa_{j}^{h} x+\Phi_{j}^{h}\right)\right]-c x+h_{0}
$$

and, realizing that $\mathrm{h}^{*}(0)=\mathrm{h}^{*}(\pi)=0, h^{*}(x)=\sum_{j=1}^{N} h_{j}^{*} \sin (j x)$ and $c=\frac{h(\pi)-h(0)}{\pi}$

where $\quad h_{j}^{*}=\frac{2}{\pi}\left[-\int_{0}^{\pi} c x \sin (j x) d x-\int_{0}^{\pi} h(0) \sin (j x) d x+\int_{0}^{\pi} \sqrt{2} \sum_{i=1}^{N} h_{i} \cos \left(\kappa_{j}^{h} x+\Phi_{j}^{h}\right) \sin (j x) d x\right]$

\section{Numerical results}

The solution of the eigenvalue problem for the $i^{\text {th }}$ sample imperfection pattern yields the critical imperfection magnitude. For each imperfection parameter, the root mean square (rms) magnitude was then combined with the critical imperfection magnitude, $\varepsilon_{\mathrm{cr}}^{(\mathrm{i})}$ to yield the rms imperfection magnitudes, $\varepsilon_{\mathrm{RMS}}^{(\mathrm{i}) \eta}$, $\varepsilon_{\text {RMS }}^{(\mathrm{i}) \mathrm{e}}, \varepsilon_{\mathrm{RMS}}^{(\mathrm{i}) \mathrm{k}}$, and $\varepsilon_{\mathrm{RMS}}^{(\mathrm{i}) \mathrm{h}^{*}}$ :

$$
\varepsilon_{R M S}^{(i) \eta}=\varepsilon_{c r}^{(i)} \sqrt{\frac{\left(\eta_{1}^{(i)}\right)^{2}+\left(\eta_{2}^{(i)}\right)^{2}}{2}} \text { and } \quad \varepsilon_{R M S}^{(i) \cdot \bullet]}=\varepsilon_{c r}^{(i)} \sqrt{\frac{1}{\pi} \int_{0}^{\pi}[\bullet(x)]^{2} d x}
$$

where [•] is e, $\mathrm{k}$, or $\mathrm{h}^{*}$ and $\mathrm{i}=1,2, \ldots, \mathrm{M}$. The product of the imperfection magnitude $\varepsilon_{\text {cr }}$ and RMS imperfection patterns should not be greater than 0.35 since it violates the nature of the perturbation approximation and are physically meaningless. Finally, the rms imperfection magnitudes are averaged over the sample size and the results tabulated. The following input parameters are used:

Sample size $=50$

Foundation stiffness: $\varphi_{0}=225$

Eccentricity standard deviation: $\sigma_{\eta}=0.05$

Imperfections simulated: $\kappa_{\mathrm{uo}}=7.449, \mathrm{~b}_{\mathrm{f} 0}=1.50, \sigma_{\mathrm{f} 0}=0.05$

Power spectral density function: $G_{f o f o}\left(\kappa_{0}\right)=\frac{1}{2} \sigma_{f o}^{2} b_{f o}^{3} \kappa_{o}^{2} \exp \left[-b_{f o}\left|\kappa_{o}\right|\right]$

Figure 1 shows the direct comparison of $\varepsilon_{\mathrm{RMS}}$ for $\eta$ alone, e alone, $\mathrm{k}$ alone, and $\mathrm{h}$ alone. Combined effects of shape and non-shape imperfections are presented in Figure 2. The first and most important observation is that the eccentricity in the applied loads is an even more dominant imperfection than that of initial shape. It was expected that eccentricity would have a similar effect upon the BEF as does initial shape since the moment it creates is a form of shape 


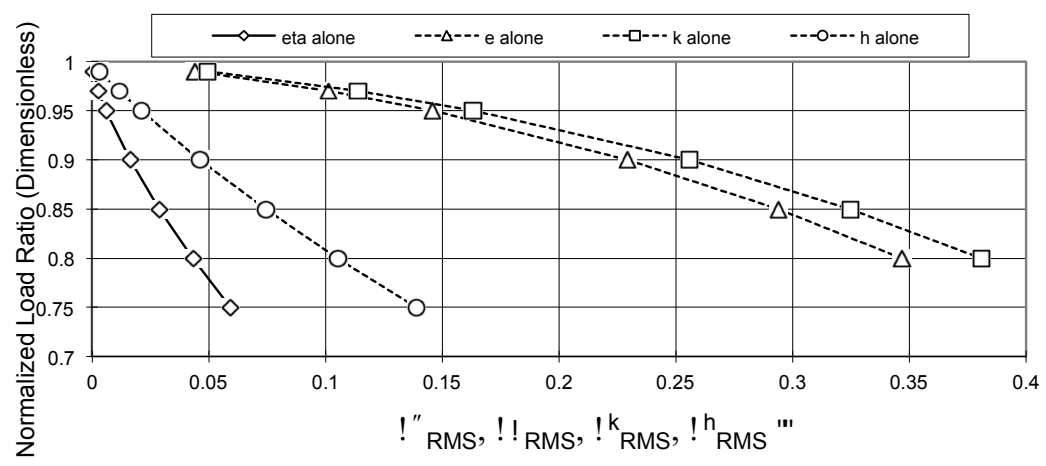

Figure 1: $\quad$ Direct comparison of e, $\mathrm{k}$, and $\mathrm{h}$.

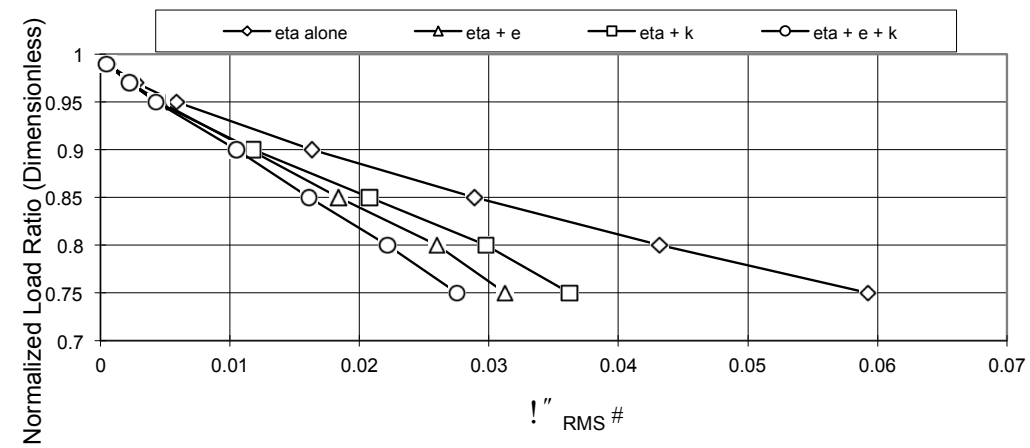

Figure 2: $\quad$ Effects of eccentricity on $\varepsilon^{\eta}$ RMS with other imperfections.

imperfection. Shape imperfections become more and more dominant as the correlation distance decreases [9].

The effects on eccentricity imperfection sensitivity will be most pronounced when it is modeled as a random variable, as it is in this case. Thus, the model used in this study maximizes the dominance of the eccentricity imperfection; while holding the dominance of shape imperfections back to an average. Eccentricity is indeed a shape imperfection. The $\varepsilon_{\text {RMS }}$ for $\eta$ are similar in shape, orientation (i.e., concave up), and location as those for $h$. This result was expected, given that the entry point of the $\eta$ s into the characteristic equation was in the $\mathrm{a}_{1 \mathrm{j}}$ term.

Figures 3 and 4 show, in the case of $\varepsilon_{\text {RMS }}$ for $\eta$ there is a remarkable twentyfold increase in sensitivity when shape imperfections are present. In the case of $\mathrm{h}$, when eccentric loads are present, the sensitivity is increased even more, by about 50 times. It is also obvious from these plots that any detrimental effects from the addition of e and $\mathrm{k}$ imperfections are so minor as to be almost inconsequential in the face of the overwhelming dominance of the two shape imperfections working in conjunction. 


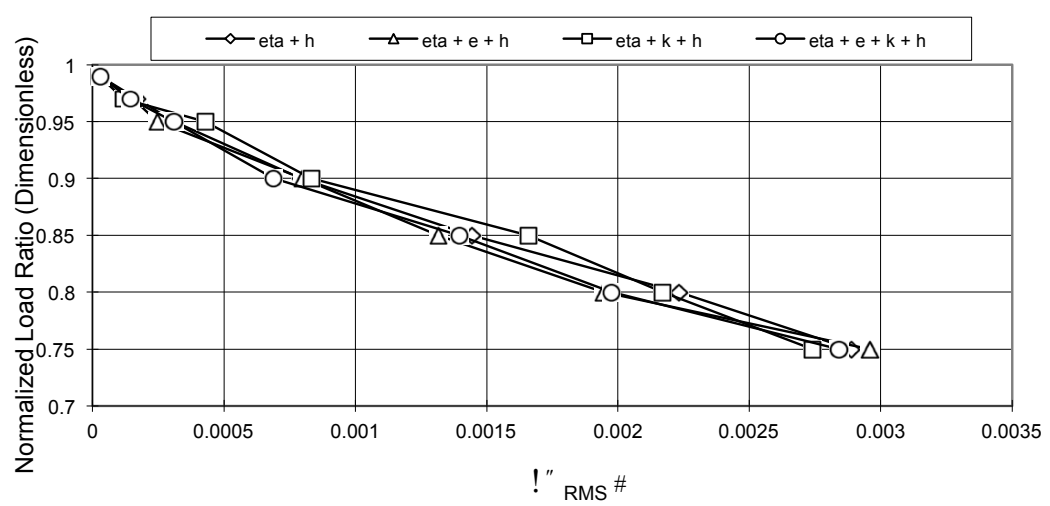

Figure 3: $\quad$ Effects of initial shape imperfections on eccentricity.

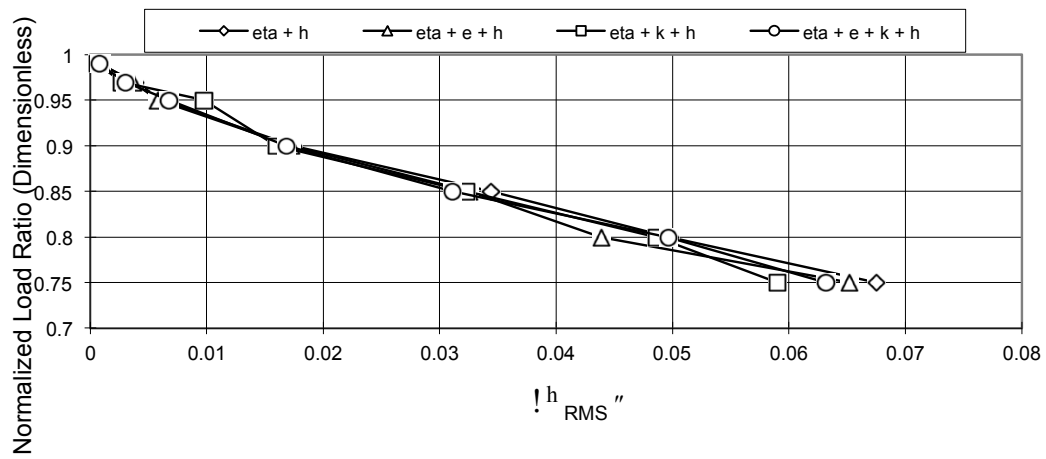

Figure 4: Effects of eccentricity on initial shape imperfections.

\section{Conclusions}

Eccentricity in the applied load is the most dominant of all imperfections for a correlation distance of 1.50. Also, the buckling sensitivity of the BEF to all other imperfections is extremely degraded in the presence of eccentricity in the applied loads. This is especially true in the case of imperfections in the initial shape of the beam. This study also proved that eccentricity in the applied load is in fact a shape imperfection. Furthermore, this study concluded that the buckling sensitivity of the beam did not depend on the sign of the product of the eccentricities on each end of the beam.

\section{References}

[1] Bazant, Z. \& Cedolin, L., Stability of Structures. Oxford, 1991.

[2] Koiter, W.T., On the Stability of Elastic Equilibrium. Ph.D. thesis. Delft, Holland, 1967. 
[3] Palassopoulos, G.V., A New approach to the Buckling of ImperfectionSensitive Structures. Journal of Engineering Mechanics, 119, pp. 850-869, 1993.

[4] Shinozuka, M. \& Deodatis, G., Simulation of Stochastic Processes by Spectral Representation. Applied Mechanics Reviews, 44(4), 1991.

[5] Thompson, J.M.T. \& Hunt, G.W., Elastic Instability Phenomena. Wiley, New York, 1984.

[6] Timoshenko, S . \& Gere, J. Mechanics of Materials, PWS-KENT, 1984.

[7] Timoshenko, S.P. \& Gere, J.M., Theory of Elastic Stability. McGraw-Hill: New York, 1961.

[8] Wilson, W.M. \& Newmark, N.M., The Strength of Thin Cylindrical Shells as Columns. Engineering Experiments Station Bulletin, U. of Ill., Urbana, Ill., 1933.

[9] Yeigh, B.W. Imperfections and Instabilities. Dissertation, Princeton, 1995. 